\title{
93. A Comparative Study on Nucleolus Organizer Regions (NORs) in 7 Rattus Species with Special Emphasis on the Organizer Differentiation and Species Evolution*)
}

\author{
By Tosihide H. YosidA \\ National Institute of Genetics, Misima \\ (Communicated by Sajiro Makino, M. J. A., Dec. 12, 1979)
}

Nucleolus organizer regions (NORs) has been recognized as an important organization in the chromosome architecture, as well as are believed to be possible sites of rRNA genes. The NORs by Ag-As staining have been demonstrated in several animals including humans (Goodpasture and Bloom 1975; Tantravahi et al. 1976, 1977; Dev et al. 1977; Bloom and Goodpasture 1976; Yosida 1978). The NORs of the black rat ( $R$. rattus) and the Norway rat ( $R$. norvegicus) have been investigated by Yosida (1978). Taxonomically the above species are closely related with apparently similar karyotypes (Yosida 1973; Yosida and Sagai 1973). They had 42 chromosomes consisting of 13 acrocentric or subtelocentric autosome pairs, 7 small metacentric autosome pairs and acrocentric $\mathrm{X}$ and $\mathrm{Y}$ chromosomes. According to Yosida (1973), the karyotype of the Norway rat was recognized as one of the polymorphic karyotypes in the Asian type black rat having 42 chromosomes. In spite of the similarity of karyotypes in the black and Norway rats, the NORs were different between two species with regard to their sites in the chromosomes (Yosida 1978).

The present paper deals with the NORs in the other 5 Rattus species in comparison with those of the black and Norway rats, with particular reference to the possible relationship between the differentiation of nucleolus organizer regions and species differentiation.

Materials and methods. Seven Rattus species used for the present study were: $R$. rattus, $R$. norvegicus, $R$. exulans, $R$. annandalei, $R$. losea, $R$. sabanus and $R$. neilli. In the black rat ( $R$. rattus) three geographical types, Asian, Oceanian and Ceylonese, and in the Norway rat four inbred strains (Wistar/Mk, Wistar/Ms, WistarKing-A and Fischer) were used. R. exulans, R. losea and R. neilli were collected from Thailand; R. annandale $i$ and $R$. sabanus from Malaysia; and then they were bred in our laboratory for about 3 to 5 generations.

*) Contribution no. 1292 from the National Institute of Genetics, Japan. Supported by a Grant-in-Aid for the Scientific Research from the Ministry of Education, Science and Culture (nos. 339025, 401001). 
Cells cultured from tail tips were exclusively used for the chromosome study. The nucleolus organizer regions were demonstrated by Ag-As technique following Goodpasture and Bloom (1975).

Results. 1) Karyotypes of 7 Rattus species. Among 7 Rattus species concerned here, 5 species ( $R$. rattus, $R$. norvegicus, $R$. exulans, $R$. annandalei and $R$. losea) had the same chromosome number $(2 n=$ 42) with similar karyotypes. Karyotypes of the former 4 species have already been reported by Yosida (1973). Their karyotypes consisted of 13 acrocentric or subtelocentric autosome pairs, 7 small metacentric autosome pairs and acrocentric $\mathrm{X}$ and $\mathrm{Y}$ chromosomes. The Asian type black rats are remarkable by acrocentric or subtelocentric polymorphism of pair nos. 1, 9 and 13, while the Norway rat was characterized by having always subtelocentric pair nos. 1,9 and $13 ; R$. exulans by having subtelocentric pair nos. 1,9 ; and $R$. annandalei by subtelocentric pair no. 9. The karyotype of $R$. losea was similar to $R$. exulans by having 42 chromosomes and pair nos. 1 and 9 subtelocentrics. The $\mathrm{X}$ and $\mathrm{Y}$ chromosomes were long and short acrocentrics similar to the other Rattus species (Fig. 1). This species seems to be

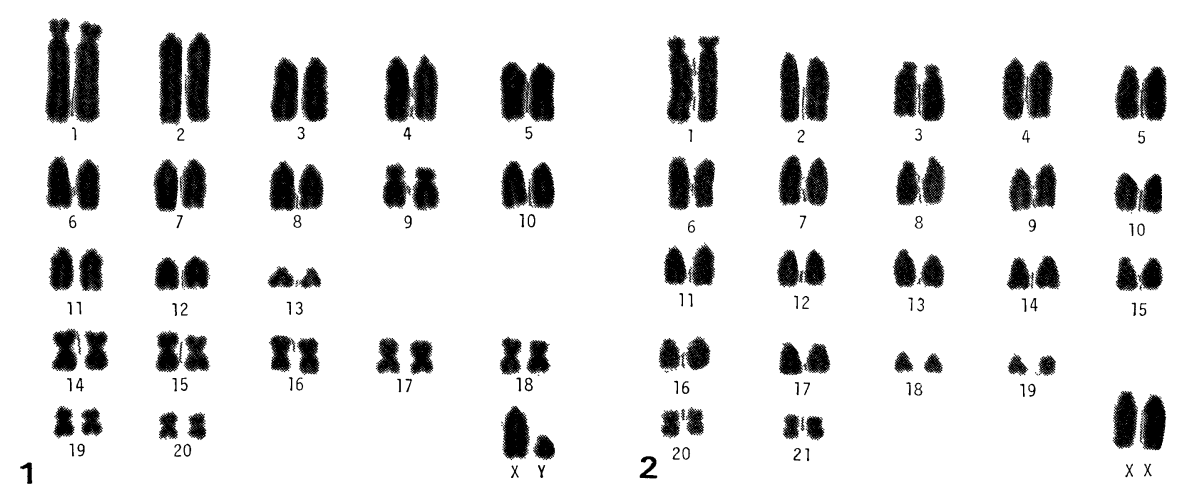

Figs. 1-2. Karyotypes of two Rattus species. 1: $R$. losea $(2 n=42)$. 2: $R$. neilli $(2 n=44)$.

classified into the first group of Rattus by the present author's classification (Yosida 1973). Rattus sabanus and $R$. neilli had very different karyotypes from the above Rattus species. In the former species the karyotypes by conventional (Yosida 1973) and banding staining (Yosida and Sagai 1973) have been reported. This rat had 42 chromosomes, among which 18 autosome pairs were acrocentrics or subtelocentrics, 2 autosome pairs were small metacentrics and $\mathrm{X}$ and $\mathrm{Y}$ chromosomes were acrocentrics. This species was classified into the third group of Rattus species by Yosida (1973). The karyotype of $R$. neilli was generally similar to the former, but the chromosome 
number was $2 n, 44$. Among 22 pairs, autosomes nos. 1 and 3 were subtelocentrics, and no. 2 and nos. 4 to 19 were acrocentrics, and the remaining two small pairs (nos. 20 and 21) metacentrics (Fig. 2). $R$. neilli was classified into the third group of Rattus species according to Yosida (1973).

2) Nucleolus organizer regions (NORs). As already reported the NORs in the black rats ( $R$. rattus) were found to occur in centromeric regions of chromosome pairs 3,8 and 13 in the following three geographical variants, Asian $(2 n=42)$, Ceylonese $(2 n=40)$ and Oceanian $(2 n=38)$ types, while in the Norway rat $(R$. norvegicus) they were found in pair nos. 3, 12 and 13 (Yosida 1978). In R. exulans the NORs were found in the centromeric regions of pair nos. 3, 5 and 13 (Fig. 3). Interestingly the NORs occurring in pair nos. 3 and 13

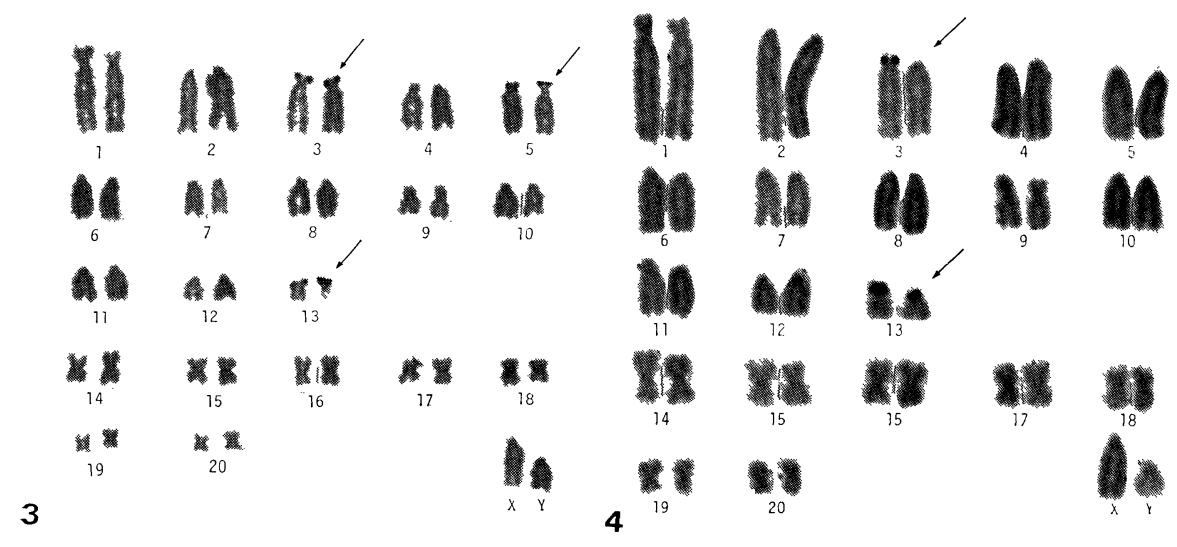

Figs. 3-4. Karyotypes showing the nucleolus organizer regions (NORs) in two Rattus species. 3: $R$. exulans (Thailand) $(2 n=42)$ with three NORs in pairs 3,5 and 13. 4: R. losea with two NORs in pairs 3 and 8 . Arrows indicate the NORs.

were common in these three Rattus species, but the other one chromosome with the NORs was different from each other. It was found in pair no. 8 for $R$. rattus, in pair no. 12 for $R$. norvegicus and in pair no. 5 for $R$. exulans.

The NORs in $R$. annandalei and $R$. losea were characterized by being located in two chromosome pairs. In the former species they were in pair nos. 8 and 13 (Fig. 5), whereas the latter species showed the NORs occurring in pair nos. 3 and 13 (Fig. 4). These chromosomes with NORs were also common, although one was less than in the above three species. The NORs in $R$. sabanus and $R$. neilli were considerably different from the above species: In the former species they were found in centromeric regions of pair nos. 1, 3 and 5 (Fig. 6), while in the latter species they occurred in only two chromosome pairs, 


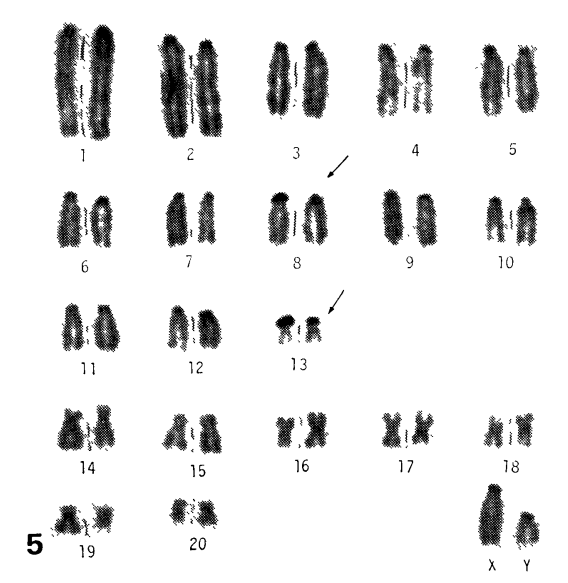

Fig. 5. Karyotype showing NORs in Rattus annandalei. The NORs are observed in pairs 8 and 13 (arrows).

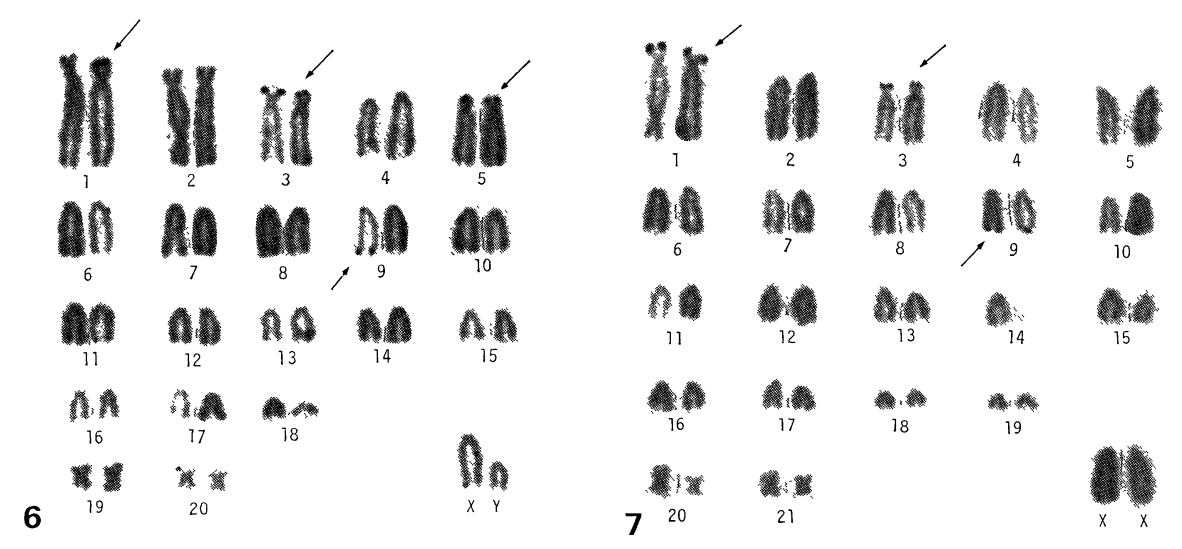

Figs. 6-7. Karyotypes showing NORs in two Rattus species belonging to the third group. 6:R. sabanus $(2 n=42)$ with four NORs in pairs $1,3,5$ and 9. 7: R. neilli with three NORs in pairs 1,3 and 9 . The NORs in pair 9 are located in telomere regions (arrows).

nos. 1 and 3 (Fig. 7). Noticeable is the fact that faintly stained NORs were found in the distal end of pair no. 9 in these two species. On the basis of the characteristics of the NORs, $R$. sabanus and $R$. neilli were considerably different from the above 5 Rattus species.

Discussion. In spite of the similarity of their karyotypes, 5 species of the first group showed a difference of the nucleolus organizer regions (NORs). The NORs were found to occur in either two or three chromosomes among pair nos. 3, 5, 8, 12 and 13. All the 5 species had the NORs in no. 13 chromosomes, and 4 of them had in pair no. 3 . In $R$. rattus another NOR occurred in pair no. 8 , the Norway rat car- 
ried it in pair no. 12 , while in $R$. exulans it was in pair no. 5. R. losea had in pair nos. 3 and 13, while $R$. annandalei showed the NORs in pair nos. 5 and 13.

The second group of Rattus species is characterized by having fewer chromosome numbers than 42 due to Robertsonian fusion from the basic karyotype (Yosida 1973). Unfortunately the NORs in the species of this group could not be examined, because of insufficient materials. The third group of Rattus species is remarkable by markedly different karyotypes from the above two Rattus groups. They were characterized by a few small metacentrics. Both of $R$. sabanus $(2 n=42)$ and $R$. neilli $(2 n=44)$ had two small metacentric pairs, in contrast to the other species having 7 small metacentric pairs. Corresponding to the karyotype differences between the first and the third groups, the NORs in the third group were markedly different from the first group. These two species, $R$. sabanus and $R$. neilli, had the NORs in pair nos. 1 and 3, but not in pair no. 13. Also they had NORs in distal ends of pair no. 9. These two species differ by the presence or absence of the NORs in pair no. 5. R. sabanus showed the NORs in this pair, whereas in $R$. neilli they were absent.

Thus the above-mentioned 7 Rattus species showed the NORs in pairs 1, 3, 5, 8, 9, 12 and 13 (Table I). Among these pairs, the NORs

Table I. Nucleolus organizer regions (NORs) in 7 Rattus species

\begin{tabular}{|c|c|c|c|c|c|c|c|c|c|}
\hline \multirow{2}{*}{ Species } & \multirow{2}{*}{$2 n$} & \multicolumn{7}{|c|}{ Chromosome pairs no. } & \multirow{2}{*}{$\begin{array}{l}\text { Total no. } \\
\text { of NORs }\end{array}$} \\
\hline & & 1 & 3 & 5 & 8 & 9 & 12 & 13 & \\
\hline \multicolumn{10}{|l|}{ R. rattus } \\
\hline Asian type & 42 & & $\bullet$ & & $\bullet$ & & & $\bullet$ & 3 \\
\hline Ceylonese type & 40 & & - & & 0 & & & $\bullet$ & 3 \\
\hline Oceanian type & 38 & & • & & - & & & - & 3 \\
\hline$R$. norvegicus & 42 & & 0 & & & & - & 0 & 3 \\
\hline R. exulans & 42 & & $\bullet$ & $\bullet$ & & & & - & 3 \\
\hline R. annandalei & 42 & & & & - & & & $\bullet$ & 2 \\
\hline$R$. losea & 42 & & ○ & & & & & $\bullet$ & 2 \\
\hline R. sabanus & 42 & 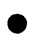 & $\bullet$ & ○ & & O & & & $3+1$ \\
\hline R. neilli & 44 & $\bullet$ & $\bullet$ & & & O & & & $2+1$ \\
\hline
\end{tabular}

Black and white circles denoted the NORs in centromere and telomere regions, respectively.

occurring in no. 3 were most frequent $(6 / 7)$, those in no. 13 ranked second $(5 / 7)$, and the NORs occurring in the other pairs were observed in one or two species. Importance is that the NORs are located in different chromosomes in each species. This feature is apparently species specific. Based on the above findings a conclusion would be 
possible that the differentiation of the nucleolus organizer regions on chromosomes is significantly related to species differentiation.

The literature refers to an interesting evidence on differentiation of the nucleolus organizer regions in relation to species evolution in man and apes (Tantravahi et al. 1976). In human chromosomes the NORs were found to occur in five following acrocentrics: nos. 13, 14, 15, 21 and 22, whereas those in chimpanzee were present in five pairs of acrocentrics which correspond to human chromosomes $12,14,18$, 21 and 22. The orangutan had the NORs in 8 acrocentric pairs, in which 6 pairs correspond to the human 13, 14, 15, 18, 21 and 22.

A question thus arises as to whether the differentiation of the NORs is dependent on cytogenetical events such as translocation, deletion and duplication, etc., or on activation and inactivation of the NORs by functional events. Miller et al. $(1976 \mathrm{a}, \mathrm{b})$, in the study of hybrid cells of mouse and human cells, have shown that the nucleolus organizer regions in human cells change by the inactive state, showing no reaction to the Ag-As staining. A the present, the cytogenetical mechanism for the differentiation of the NORs remains unknown in the genus Rattus. Cytogenetical events involving translocation, deletion, duplication, etc. seem to be essential for differentiation of the nucleolus organizer regions.

Acknowledgments. The author is very grateful to Emeritus Professor Sajiro Makino, M. J. A., for his revision of this manuscript, to Mrs. Y. Ochiai-Hirai for technical assistance, and to Dr. B. B. Parida for the preparation of this manuscript.

\section{References}

Bloom, S. E., and Goodpasture, C.: Hum. Genet., 34, 199-206.

Dev, V. G., Tantravahi, R., Miller, D. A., and Miller, O. H.: Genetics, 86, 389398.

Goodpasture, C., and Bloom, E.: Chromosoma (Berl.), 53, 37-50 (1975).

Miller, D. A., Dev, V. G., Tantravahi, R., and Miller, O. J.: Exp. Cell Res., 101, 235-243 (1976a).

Miller, O. J., Miller, D. A., Dev, V. G., Tantravahi, R., and Croce, C. M.: Proc. Nat. Acad. Sci. (U.S.), 73, 4531-4535 (1976b).

Tantravahi, R., Miller, D. A., Dev, V. G., and Miller, O. H.: Chromosoma (Berl.), 56, 15-27 (1976).

Tantravahi, R., Miller, D. A., and Miller, O. J.: Cytogenet. Cell Genet., 18, 364369 (1977).

Yosida, T. H.: Chromosoma (Berl.), 40, 285-297 (1973).

—-: Proc. Japan Acad., 54B, 353-358 (1978).

Yosida, T. H., and Sagai, T.: Chromosoma (Berl.), 41, 93-101 (1973). 